\title{
Revista Iberoamericana de Automática e Informática Industrial
}

Revista Iberoamericana de Automática e Informática Industrial 19 (2022) 13-26

\section{Integración de la estrategia FMBPC en una estructura de control predictivo en lazo cerrado. Aplicación al control de fangos activados}

\author{
Pedro M. Vallejo*, Pastora Vega \\ Grupo de Supervisión y Control de Procesos, Dpto. de Informática y Automática, Universidad de Salamanca, \\ Facultad de Ciencias, Plaza de los Caídos, s/n, 37008, Salamanca, España.
}

To cite this article: Vallejo, P.M., Vega, P. 2022. Integration of the FMBPC strategy in a closedloop predictive control structure. Application to the control of activated sludge. Revista Iberoamericana de Automática e Informática Industrial 19, 13-26. https://doi.org/10.4995/riai.2021.15793

\begin{abstract}
Resumen
En este trabajo se aborda la integración de dos métodos o estrategias de Control Predictivo basado en Modelos, a saber: Control Predictivo basado en Modelos Borrosos (FMBPC) y Control Predictivo en Lazo Cerrado (CLP-MPC). La primera de estas estrategias utiliza principios de Control Predictivo Funcional (PFC) y está enmarcada, al mismo tiempo, en el ámbito del Control Inteligente (IC). La integración tiene como principal objetivo proporcionar a la estrategia de control no lineal FMBPC un procedimiento de optimización que permita el manejo automático de restricciones en la variable de control. La solución propuesta consiste en hacer uso de una estructura complementaria de tipo CLP-MPC para determinar mediante optimización, en cada instante de muestreo, los valores óptimos de un cierto término aditivo, a sumar a la ley de control FMBPC, de tal modo que se satisfagan las restricciones. El modelo de predicciones y la ley de control base necesarios para realizar los cálculos en la estructura CLP-MPC son proporcionados por la estrategia FMBPC. La estrategia mixta FMBPC/CLP propuesta ha sido validada, en simulación, aplicándola al control de fangos activados en plantas de tratamiento de aguas residuales (EDAR), poniendo el foco en la imposición de restricciones a la acción de control. Los resultados obtenidos son satisfactorios, observando un buen rendimiento del algoritmo de control diseñado, al tiempo que se garantiza tanto la satisfacción de las restricciones, que era el principal objetivo, como la estabilidad del sistema en lazo cerrado.
\end{abstract}

Palabras clave: Control predictivo basado en modelo, control borroso y sistemas borrosos en control, técnicas de control inteligente, control de sistemas con restricciones, control multivariable, control automático de sistemas de tratamiento de aguas.

Integration of the FMBPC strategy in a closed-loop predictive control structure. Application to the control of activated sludge.

\begin{abstract}
This work addresses the integration of two methods or strategies of Model-Based Predictive Control, namely: Fuzzy Model-Based Predictive Control (FMBPC) and Closed-Loop Predictive Control (CLP-MPC). The first of these strategies uses principles of Predictive Functional Control (PFC) and is framed, at the same time, in the field of Intelligent Control (IC). The main objective of the integration is to provide to the FMBPC nonlinear control strategy an optimization procedure that allows the automatic handling of constraints in the control variable. The proposed solution consists of making use of a complementary structure of the CLP-MPC type to determine by optimization, at each sampling instant, the optimal values of a certain additive term, to be added to the FMBPC control law, in such a way that they are satisfied the constraints. The prediction model and base control law necessary to perform the calculations on the CLP-MPC structure are provided by the FMBPC strategy. The proposed FMBPC/CLP mixed strategy has been validated, in simulation, applying it to the control of activated sludge processes in wastewater treatment plants (WWTP), focusing on the imposition of constraints on the control action. The results obtained are satisfactory, observing a good performance of the designed control algorithm, while guaranteeing both the satisfaction of the constraints, which was the main objective, and the stability of the closed-loop system.
\end{abstract}

Keywords: Model-based predictive control, fuzzy control and fuzzy systems in control, intelligent control techniques, control of systems with restrictions, multivariable control, automatic control of water treatment systems. 


\section{Introducción}

La estrategia de control predictivo basado en modelos borrosos o, mediante su correspondiente expresión en inglés, Fuzzy Model-based Predictive Control (FMBPC) (Babuška, 1998a; Roubos et al., 1999; Mollov et al., 2004; Blažič et al., 2007; Bououden et al., 2015; Škrjanc et al., 2016; Boulkaibet et al., 2017; Vallejo et al., 2019, 2021), constituye una modalidad de control predictivo no lineal idónea para controlar de manera eficaz sistemas altamente no lineales o con incertidumbres $\mathrm{o}$, en general, sistemas con dinámicas complejas, como por ejemplo los sistemas biológicos. Tal capacidad deriva de la utilización de un modelo de predicciones borroso (fuzzy model) (Zadeh, 1990), generalmente obtenido mediante identificación a partir de datos experimentales de entrada-salida. Si la identificación borrosa (Babuška, 1998b) está bien diseñada, será capaz de capturar la dinámica de la planta de manera fiel, lo cual resulta determinante en la fiabilidad de las predicciones y, en última instancia, en el rendimiento del controlador predictivo. Esta propiedad puede ser identificada como la componente o faceta de la estrategia de control FMBPC que la sitúa (parcialmente) también en el ámbito del control inteligente (IC), puesto que está relacionada con la utilización de razonamiento cualitativo, presente en el modelado borroso. Se trata, por tanto, de un controlador que pertenece, tanto a la familia de los controladores predictivos no lineales, como a la de los controladores inteligentes. En el contexto del presente trabajo, la estrategia FMBPC considerada se enmarca además en el ámbito del denominado control predictivo funcional o (en inglés) Predictive Functional Control (PFC), que utiliza el denominado principio de equivalencia (Richalet, 1993; Škrjanc et al., 2000; Richalet et al., 2009; Haber et al., 2016). El principio de equivalencia establece en esencia que, dado un cierto proceso y dado un modelo perfecto del mismo, el incremento en la salida del proceso en respuesta a un determinado incremento de la acción de control deberá ser equivalente al incremento en la salida del modelo para el mismo cambio en la acción de control. Esto es utilizado en PFC en el tratamiento matemático correspondiente a los denominados puntos de coincidencia de la trayectoria de referencia (véase también: Vallejo et al., 2019).

En (Vallejo et al., 2019, 2021), la estrategia FMBPC fue aplicada al control multivariable de procesos biológicos de depuración de aguas residuales mediante fangos activados, obteniendo buenos resultados. Sin embargo, en tales propuestas no fueron implementados procedimientos de manejo automático de restricciones en la acción de control, o en otras variables, sujetos a optimización (Maciejowski, 2002; Limón, 2002). La incorporación de un procedimiento de ese tipo para el manejo de las restricciones en la variable de control (en cada iteración de la implementación online del control predictivo) es precisamente una de las principales razones y motivaciones del presente trabajo.

La solución propuesta en el presente trabajo se basa en la combinación de la estrategia FMBPC con una determinada estructura de control predictivo basado en modelos (MPC) (Camacho et al., 1998), denominada paradigma de lazo cerrado o, conforme a su definición original en inglés, Closed-Loop Paradigm (CLP), conocida también como control predictivo en lazo cerrado (Rossiter, 2003). El control CLP es en realidad una modalidad del denominado control predictivo en modo dual o Dual-Mode Control (DM-MPC) (Michalska et al., 1993; Rossiter, 2003), que más que una estrategia propiamente dicha, es una manera particular de organizar el conjunto de las predicciones, dividiéndolo en dos horizontes o etapas, en cada una de las cuales se computan de manera diferente las acciones de control futuras. Esta configuración facilita el diseño de algoritmos de control MPC; con estabilidad garantizada y buen rendimiento. En el caso de la modalidad de control en modo dual de tipo CLP, a la que denominaremos en adelante CLP-MPC, en cada uno de los pasos del primer tramo de las predicciones (modo 1) se sustituye la variable de entrada manipulada por la expresión correspondiente a una ley de control estabilizante, previamente elegida, más un término complementario dependiente del tiempo y sujeto a optimización, denominado perturbación de la acción de control (al que representaremos en adelante como $c_{j}$, en referencia al paso $j$-ésimo, o como $c$, de forma genérica). En el segundo tramo (modo 2), las predicciones se computan sustituyendo la entrada manipulada únicamente por la expresión correspondiente a la ley de control estabilizante elegida. Los elementos de la secuencia de perturbaciones de la acción de control constituyen los grados de libertad del problema de optimización y sus valores óptimos se obtendrán mediante minimización de una determinada función de coste. El primer valor de la secuencia óptima obtenida será el elegido para la implementación de la acción de control online, en el periodo de muestreo correspondiente. La estructura CLP-MPC tiene las ventajas propias de la estructura DM-MPC (dado que es un caso particular de esta) y constituye un método útil para mejorar algunos aspectos importantes en procedimientos de optimización en tiempo real, ligados a estrategias de control predictivo MPC, como el acondicionamiento numérico y la carga computacional (reduciéndola), o el análisis de estabilidad o de robustez (Adetola et al., 2010; Marchetti et al., 2014).

Algunos trabajos relativamente recientes en los que se utilizan las estructuras de control predictivo DM-MPC o CLP-MPC pueden verse en: (Ramírez et al., 2014), (Shariati et al., 2015), (El Bahja et al., 2018a, 2018b). Y enmarcados en el ámbito del control predictivo distribuido, en: (Al-Gherwi et al., 2013), (Sorcia-Vázquez et al., 2015). La reducción de la carga computacional en los cálculos de optimización asociados a estas estructuras hace especialmente interesante su uso en control predictivo distribuido.

En relación con los métodos de diseño de las estructuras de control predictivo CLP-MPC, en (Rossiter, 2003) se propone utilizar como ley de control base estabilizante una ley de realimentación de estados estándar del tipo $u=-K x$ y al mismo tiempo se sugiere la posibilidad de que dicha ley pueda ser sustituida por otra. En (El Bahja et al., 2018b) se propone una metodología que utiliza conjuntos invariantes poliédricos y se deduce una ley de control CLP-MPC de realimentación de estados, estabilizante y que asegura el cumplimento de las restricciones y de los requisitos de rendimiento del sistema operando en lazo cerrado. En (El Bahja et al., 2018a) se propone como ley de control base estabilizante de la estructura CLP-MPC una ley de Control Predictivo Generalizado no lineal (NLGPC), que será expresada de forma analítica, y se 
utiliza un modelo fenomenológico de la planta para el cálculo de las predicciones (formalizado como un modelo lineal con coeficientes dependientes del estado). Sin embargo, el uso de modelos fenomenológicos restringe la aplicabilidad de estas técnicas debido a la dificultad, tanto de la obtención, como del manejo de tal tipo de modelos. Como alternativa, se plantea la utilización de modelos basados en datos, más fáciles de obtener y de manejar.

Así, en el presente trabajo se utilizan modelos basados en datos numéricos de entrada-salida, obtenidos mediante técnicas de identificación borrosa, capaces de capturar fielmente la dinámica de la planta. La identificación borrosa produce como resultado, originariamente, modelos borrosos discretos de tipo Takagi-Sugeno (TS) (Takagi et al., 1985), pero después estos son convertidos en (Vallejo et al., 2019, 2021) en modelos equivalentes en el espacio de estados, lineales y variantes en el tiempo, es decir (mediante sus siglas en inglés) de tipo DLTV (discrete linear time-varying), a escala global, y en modelos lineales e invariantes en el tiempo, es decir (en inglés) de tipo DLTI (discrete linear time-invariant), a escala local. Concretamente, estos últimos serán los que se emplearán como modelos de predicciones dentro de la estructura CLP-MPC, correspondiendo un modelo DLTI diferente a cada período de muestreo.

La estrategia mixta de control predictivo presentada en este artículo consiste, en esencia, en considerar la estrategia FMBPC como la principal y utilizar de modo complementario la estructura CLP-MPC, en el marco de la cual se determinarán mediante optimización los valores óptimos de las perturbaciones de la acción de control (términos $c$ ), en cada período de muestreo. En el cálculo de predicciones correspondiente a la estructura CLP-MPC se utilizará como ley de control base estabilizante la ley correspondiente a la estrategia FMBPC, propuesta y desarrollada en (Vallejo et al., 2019, 2021), que puede considerarse matemáticamente equivalente a una ley de control del tipo $u=-K x$, con $K$ dependiente del tiempo. En relación con el procedimiento de optimización, este consistirá en minimizar una cierta función de coste, dependiente de los grados de libertad del problema, es decir, de las variables $c$ (estrictamente, $c_{j}$ ), con sujeción al cumplimiento de las restricciones previamente establecidas, obteniéndose así la secuencia óptima de tales términos. El primer elemento de esa secuencia será sumado online al valor calculado mediante la ley FMBPC, obteniéndose así el valor efectivo de la acción de control, a aplicar a la planta en el instante de muestreo $k$-ésimo en curso (instante de muestreo actual), con la forma equivalente siguiente: $u=-K x+c$. En relación con las restricciones, en nuestro caso se ha puesto el énfasis en la limitación de los valores de la acción de control.

Las principales características de la estrategia de control predictivo no lineal propuesta, a la que denominaremos FMBPC/CLP, son: la utilización de modelos borrosos no lineales, obtenidos a partir de datos numéricos de entrada-salida y la aplicación de una ley de control predictivo del tipo FMBPC, no lineal, analítica y explícita, que asegura la estabilidad local del sistema en lazo cerrado y que, con la ayuda de la estructura complementaria CLP-MPC, asegura también al mismo tiempo el cumplimiento de las restricciones.

En relación con las aportaciones de la estrategia FMBPC/CLP propuesta, podemos separarlas por ámbitos. En el ámbito de las estrategias FMBPC con leyes de control analíticas y explícitas, nuestra estrategia incorpora un mecanismo automático de imposición de restricciones. En el ámbito del control predictivo, una aportación importante es la integración de dos metodologías de control predictivo diferentes, como son el control predictivo PFC, utilizado en la estrategia FMBPC considerada y el control predictivo clásico, basado en la optimización de funciones de coste, que es el método utilizado en la estructura CLP-MPC para determinar las acciones de control óptimas. Pero además, la combinación de esas dos metodologías (FMBPC y CLP-MPC) produce, como resultado, una nueva estrategia de MPC no lineal, con ciertas características de control inteligente en la identificación del modelo y con una importante reducción de la carga computacional en el manejo de restricciones, en comparación con otras metodologías de MPC no lineal en lazo abierto. Y finalmente, en el ámbito de las estrategias basadas en la estructura CLP-MPC, la estrategia presentada utiliza modelos obtenidos mediante identificación borrosa, a partir de datos numéricos, en lugar de modelos fenomenológicos, con las consiguientes ventajas en cuanto a la obtención, fidelidad y manejo del modelo (como ya se indicó anteriormente). Así mismo, desde una perspectiva más global y genérica, la metodología desarrollada puede constituir un posible marco para el diseño de leyes de control de procesos no lineales, con múltiples entradas y salidas y restricciones en las entradas, dotado con capacidades explícitas de manejo de restricciones y de caracterización de las propiedades de estabilidad y desempeño del controlador, en la fase de diseño de este.

La estrategia de control desarrollada se ha validado aplicándola al control de procesos de fangos activados en estaciones depuradoras de aguas residuales, que es la planta que ha sido tomada como caso de estudio.

El resto del artículo está estructurado de la forma siguiente: en la sección 2 se resumen los fundamentos y la base matemática del control predictivo en modo dual (brevemente) y de la estructura CLP en particular; en la sección 3 se revisa la estrategia de control predictivo FMBPC considerada, enfocada al control de fangos activados; en la sección 4 se explican los fundamentos de la propuesta de integración de la estrategia FMBPC con una estructura de control predictivo CLP-MPC, con el objetivo de manejar las restricciones en la acción de control; en la sección 5 se describen los experimentos de simulación y se muestran y se analizan los resultados; y por último, en la sección 6 se detallan las conclusiones.

\section{Control predictivo en lazo cerrado}

La estructura CLP-MPC es, como se ha dicho, un caso particular de estructura de control predictivo en modo dual (DM-MPC). Resumimos a continuación los fundamentos y la base matemática necesarias (Rossiter, 2003; El Bahja, 2017).

Representación de la planta. Supondremos que la planta en lazo abierto podrá representarse mediante un modelo lineal en el espacio de estados, es decir:

$$
\begin{gathered}
\boldsymbol{x}_{k+1}=A \boldsymbol{x}_{k}+B \boldsymbol{u}_{k} \\
\boldsymbol{y}_{k}=C \boldsymbol{x}_{k}
\end{gathered}
$$


siendo $\boldsymbol{x}_{k}$ el estado en el instante $k$-ésimo, $\boldsymbol{u}_{k}$ las entradas manipulables e $\boldsymbol{y}_{k}$ las salidas medibles de la planta.

Por otra parte, en general asumiremos como ley de control en lazo cerrado la conocida ley de realimentación de estados:

$$
\boldsymbol{u}_{k}=-K \boldsymbol{x}_{k}
$$

siendo $K$ un vector o matriz con las dimensiones adecuadas y definiremos:

$$
\Phi=(A-B K)
$$

Control predictivo en modo dual. La estructura DM-MPC original, también llamada paradigma en lazo abierto (OLP), consiste en considerar el horizonte de predicción dividido en dos tramos diferentes o modos (modo 1 y modo 2), con diferentes tipos de acción de control para cada uno: para los $n_{c}$ primeros pasos (modo 1), correspondientes a estados supuestamente lejanos al punto de operación deseado, las acciones de control, $\boldsymbol{u}_{k+j \mid k}\left(j=0,1, \ldots, n_{c}-1\right)$, se consideran libres, a determinar mediante optimización, y para el resto de pasos, a partir de $n_{c}+1$ (modo 2 ), correspondientes a estados supuestamente más cercanos ya al punto de operación, las acciones de control vendrán dadas por una ley de control en lazo cerrado previamente fijada (muy habitualmente, del tipo $-K \boldsymbol{x}_{k+n_{c}+j \mid k}$, con $j=0,1, \ldots, n_{y}-n_{c}-1$, para un horizonte de predicción de $n_{y}$ pasos). La secuencia de acciones de control predichas para todo el horizonte de predicción (en el instante de muestreo $k$ ) será una matriz a la que denotaremos con $\boldsymbol{u}_{k(O L P-M P C)}$. Las primeras $n_{c}$ componentes de esta matriz (modo 1) constituyen los grados de libertad $o$, mediante sus siglas en inglés, d.o.f. (degrees of freedom) de esta configuración y son determinados mediante optimización, típicamente buscando el mínimo de una cierta función de coste, sin restricciones o con ellas. Esta configuración es tenida en cuenta únicamente para las predicciones. Para la implementación online en cada período de muestreo, se toma el primer valor de la secuencia óptima de acciones de control obtenida.

Estabilidad garantizada en modo dual. En (Rossiter, 2003) se analiza la estabilidad de sistemas de control predictivo en modo dual, estableciendo una conclusión importante: el uso de horizontes infinitos y la inclusión en las predicciones de la denominada cola (tail, en inglés) permiten demostrar que la función de coste asociada al problema de optimización, (a la que denotaremos con, $\left.J_{k(D M)}\right)$, es una función de Lyapunov y por tanto puede concluirse que la ley de control DM-MPC será estable, según la teoría de estabilidad de Lyapunov (Lyapunov, 1892, 1992).

Control predictivo en lazo cerrado. La modalidad del control predictivo en modo dual CLP-MPC consiste en considerar las acciones de control del modo 1, durante las predicciones, como suma de dos términos: un primer término correspondiente a una ley de control en lazo cerrado previamente fijada (típicamente, la misma que en el modo 2) y un segundo término, o término complementario, $\boldsymbol{c}_{k}$, con el rol de grado de libertad, sujeto a optimización, y cuyo objetivo sea el manejo de las restricciones. En el caso de que la ley de control preestablecida esté basada en una realimentación de estados, $-K \boldsymbol{x}$, el modelo de predicciones se expresará como se especifica (a continuación) en la ecuación (4). Los términos $\boldsymbol{c}_{k}$ son conocidos como perturbations (perturbaciones de la acción de control), lo cual no debe confundirse con el concepto estándar de perturbaciones en la entrada, o en el estado, nombrado en la literatura de control automático en inglés como disturbances). En el modo 2 de la estructura CLP-MPC, las acciones de control predictivas serán las correspondientes a la ley de control en lazo cerrado previamente fijada, tal y como se muestra (a continuación) en la ecuación (5):

$$
\begin{aligned}
& \text { Modo } 1 \text { (CLP-MPC) } \\
& \boldsymbol{x}_{k+i+1 \mid k}=A \boldsymbol{x}_{k+i \mid k}+B\left(-K \boldsymbol{x}_{k+i \mid k}+\boldsymbol{c}_{k+i \mid k}\right) \\
& =\Phi \boldsymbol{x}_{k+i \mid k}+B \boldsymbol{c}_{k+i \mid k} \\
& \boldsymbol{u}_{k+i \mid k}=-K \boldsymbol{x}_{k+i \mid k}+\boldsymbol{c}_{k+i \mid k}
\end{aligned}
$$

siendo: $c_{k+i \mid k}$ :d.o.f, $i=0,1,2, \ldots, n_{c}-1$

\section{Modo 2 (CLP-MPC)}

$$
\begin{gathered}
\boldsymbol{x}_{k+i+1 \mid k}=A \boldsymbol{x}_{k+i \mid k}+B\left(-K \boldsymbol{x}_{k+i \mid k}\right) \\
=\Phi \boldsymbol{x}_{k+i \mid k} \\
\boldsymbol{u}_{k+i \mid k}=-K \boldsymbol{x}_{k+i \mid k}
\end{gathered}
$$

siendo: $i>n_{c}$

Y teniendo en cuenta ahora las ecuaciones (4) y (5) y mediante el oportuno desarrollo, la expresión matricial de la secuencia de acciones de control predichas en el instante de muestreo $k$ correspondientes a la estructura CLP-MPC, a la que denotaremos con $\boldsymbol{u}_{k(C L P-M P C)}$, quedará como sigue:

$$
\boldsymbol{u}_{k(C L P-M P C)_{\rightarrow}}=\left[\begin{array}{c}
-K \boldsymbol{x}_{k \mid k}+\boldsymbol{c}_{k \mid k} \\
-K \boldsymbol{x}_{k+1 \mid k}+\boldsymbol{c}_{k+1 \mid k} \\
\vdots \\
-K \boldsymbol{x}_{k+n_{c}-1 \mid k}+\boldsymbol{c}_{k+n_{c}-1 \mid k} \\
------ \\
-K \boldsymbol{x}_{k+n_{c} \mid k} \\
-K \Phi \boldsymbol{x}_{k+n_{c} \mid k} \\
\vdots \\
-K \Phi^{n_{y}-n_{c}-1} \boldsymbol{x}_{k+n_{c} \mid k}
\end{array}\right]
$$

siendo $\operatorname{los} n_{c}$ términos complementarios del modo 1 los grados de libertad del problema de optimización, que pueden ser expresados en forma matricial de la forma siguiente:

$$
\text { d. o. } \boldsymbol{f}_{k(C L P-M P C) \rightarrow}=\boldsymbol{c}_{k(C L P-M P C) \rightarrow}=\left[\begin{array}{c}
\boldsymbol{c}_{k \mid k} \\
\boldsymbol{c}_{k+1 \mid k} \\
\vdots \\
\boldsymbol{c}_{k+n_{c}-1 \mid k}
\end{array}\right]
$$

Haciendo uso de las ecuaciones (4), (5), (6) y (7) y mediante el apropiado desarrollo, es posible obtener las expresiones matriciales correspondientes al conjunto de las predicciones en todo el horizonte de predicción, que lógicamente dependerán de las variables d.o.f, $\boldsymbol{c}_{k(C L P-M P C) \rightarrow}$. Así mismo, una vez que haya sido elegida la función de coste $J_{k(C L P)}$ y que hayan sido especificadas las restricciones, es posible también formalizar sus correspondientes expresiones en función de $\boldsymbol{c}_{k(C L P-M P C)_{\rightarrow}}$. 
A partir de ello podrá expresarse el problema de optimización en función de las variables d.o.f, lo cual conducirá a la determinación de la acción de control óptima (o incremento óptimo) para cada instante $k$. A continuación se detallan las expresiones más relevantes consideradas y asumidas (comenzando por un cambio de notación con objetivos de simplificación).

Simplificación de la notación. Con el objetivo de mejorar la comprensión matemática, se utilizará la siguiente notación (donde se ha considerado también la secuencia de los estados $\boldsymbol{x}_{k}$ a lo largo del horizonte de predicción, $\left.\boldsymbol{x}_{k(C L P-M P C)_{\rightarrow}}\right)$ :

$$
\begin{aligned}
\boldsymbol{u}_{k(C L P-M P C)_{\rightarrow}} & \equiv \boldsymbol{u}_{\rightarrow} \\
\boldsymbol{c}_{k(C L P-M P C)_{\rightarrow}} & \equiv \boldsymbol{c}_{\rightarrow} \\
\boldsymbol{x}_{k(C L P-M P C)_{\rightarrow}} & \equiv \boldsymbol{x}_{\rightarrow}
\end{aligned}
$$

Predicciones correspondientes a la estructura CLP-MPC. Las expresiones finales correspondientes al estado, $\boldsymbol{x}_{\rightarrow}$, y a las acciones de control, $\boldsymbol{u}_{\rightarrow}$, son mostradas a continuación (siendo $A$ y $B$ las matrices del modelo lineal dado por la ecuación (1) y $\Phi$ la expresión definida en (3)):

$$
\boldsymbol{x}_{\rightarrow}=P_{c l} \boldsymbol{x}_{k}+H_{c} \boldsymbol{c}_{\rightarrow}
$$

donde:

$$
\begin{aligned}
& \begin{array}{llllll}
\Phi & B & 0 & 0 & \cdots
\end{array} \\
& \begin{array}{lllll}
\Phi^{2} & \Phi B & B & 0 & \cdots
\end{array} \\
& P_{c l}=\Phi^{3}, \quad H_{c}=\Phi^{2} B \quad \Phi B \quad B \quad \cdots \\
& \lfloor\rfloor\left\lfloor\left\lfloor\begin{array}{llll}
\vdots & \vdots & \vdots & \vdots
\end{array}\right.\right. \\
& \boldsymbol{u}_{\rightarrow}=P_{c l u} \boldsymbol{x}_{k}+H_{c u} \boldsymbol{c}_{\rightarrow}
\end{aligned}
$$

donde:

$$
\begin{aligned}
& P_{c l u}=\left[\begin{array}{c}
-K \\
-K \Phi \\
-K \Phi^{2} \\
\vdots \\
\end{array}\right] \\
& H_{c u}=\begin{array}{cccc}
B & 0 & 0 & \cdots \\
-K B & B & 0 & \cdots \\
-K \Phi B & -K B & B & \cdots \\
\vdots & \vdots & \vdots & \vdots
\end{array}
\end{aligned}
$$

En principio, las expresiones contenidas en las ecuaciones (9) y (10) serían sólo las correspondiente al modo 1 y por lo tanto las dimensiones de $\boldsymbol{x}_{\rightarrow}$ y de $\boldsymbol{u}_{\rightarrow}$, así como las de las matrices $P_{c l}, H_{c}, P_{c l u}$ y $H_{c u}$, dependerían del número de pasos del modo 1 , es decir, de $n_{c}$. Sin embargo, es posible expresar las acciones de control del modo 2 de la misma forma que en el modo 1, es decir: $\boldsymbol{u}_{k+i \mid k}=-K \boldsymbol{x}_{k+i \mid k}+\boldsymbol{c}_{k+i \mid k}$, imponiendo $\boldsymbol{c}_{k+i \mid k}=0$, para todo $i \geq n_{c}$. Esto es equivalente a extender $\boldsymbol{c}_{\rightarrow}$ a todo el horizonte de predicción, de tal manera que todas las componentes a partir de la $n_{c}$-ésima sean nulas. En tal supuesto, las ecuaciones (9) y (10) pueden representar a las predicciones en ambos modos. En la frontera de separación entre ambos modos, es decir, en el paso $\left(n_{c}+1\right)$, la expresión del estado predicho, $\boldsymbol{x}_{k+n_{c} \mid k}$, puede deducirse de (9), obteniéndose lo siguiente:

$$
\boldsymbol{x}_{k+n_{c} \mid k}=P_{c l 2} \boldsymbol{x}_{k}+H_{c 2} \boldsymbol{c}_{\rightarrow}
$$

siendo $P_{c l 2}$ y $H_{c 2}$ las submatrices de $P_{c l}$ y $H_{c}$, respectivamente, correspondientes a la fila $\left(n_{c}+1\right)$-ésima de $\boldsymbol{x}_{\rightarrow}$. La razón por la que se ha detallado la expresión correspondiente al estado $\boldsymbol{x}_{k+n_{c} \mid k}$ (ecuación (11)) es que las acciones de control predichas para el modo 2 dependen todas de tal estado (véase la ecuación (6)).

Finalmente, cabe observar y resaltar que las expresiones de las predicciones dadas por (9) y (10), es decir, las correspondientes a los estados $\boldsymbol{x}_{\rightarrow}$ y a las acciones de control $\boldsymbol{u}_{\rightarrow}$, tienen, ambas, forma de combinación lineal o función afín de $\boldsymbol{x}_{k}$ y $\boldsymbol{c}_{\rightarrow}$, lo cual facilita la computación.

Función de coste $J_{k(C L P)}$. Asumiremos como función de coste la expresión siguiente (habitual en control predictivo):

$$
\begin{aligned}
J_{k(C L P)}= & \sum_{i=0}^{\infty}\left(\boldsymbol{x}_{k+i+1}^{T} Q \boldsymbol{x}_{k+i+1}+\boldsymbol{u}_{k+i}^{T} R \boldsymbol{u}_{k+i}\right) \\
= & \sum_{i=0}^{n_{c}-1}\left(\boldsymbol{x}_{k+i+1}^{T} Q \boldsymbol{x}_{k+i+1}+\boldsymbol{u}_{k+i}^{T} R \boldsymbol{u}_{k+i}\right) \\
& +\sum_{i=n_{c}}^{\infty}\left(\boldsymbol{x}_{k+i+1}^{T} Q \boldsymbol{x}_{k+i+1}+\boldsymbol{u}_{k+i}^{T} R \boldsymbol{u}_{k+i}\right)
\end{aligned}
$$

donde los parámetros de ponderación $Q$ y $R$ son matrices definidas positivas y constituyen parámetros de sintonía en las estrategias de control predictivo. Llevando ahora a cabo el oportuno desarrollo y las consideraciones necesarias, la expresión (12) puede ser formalizada de la forma siguiente:

$$
J_{k(C L P)}=\boldsymbol{c}_{\rightarrow}^{T} S_{C} \boldsymbol{c}_{\rightarrow}+2 \boldsymbol{c}_{\rightarrow}^{T} S_{c x} \boldsymbol{x}_{\boldsymbol{k}}+k
$$

donde:

$$
\begin{gathered}
S_{c}=H_{c}^{T} \operatorname{diag}(Q) H_{c}+H_{c u}^{T} \operatorname{diag}(R) H_{c u} \\
+H_{c 2}^{T} P H_{c 2} \\
S_{c x}=H_{c}^{T} \operatorname{diag}(Q) P_{c l}+H_{c u}^{T} \operatorname{diag}(R) P_{c l u} \\
+H_{c 2}^{T} P P_{c l 2}
\end{gathered}
$$

siendo posible ignorar el término $k$ (el tercer sumando de la expresión de $J_{k(C L P)}$ ), dado que es independiente de $\boldsymbol{c}_{\rightarrow}$ y que la optimización se planteará respecto de $\boldsymbol{c}_{\rightarrow}$. Por tanto, podemos tomar como función $J_{k(C L P)}$ la expresión siguiente (equivalente a (13), en cuanto al procedimiento de optimización se refiere):

$$
J_{k(C L P)}=\boldsymbol{c}_{\rightarrow}^{T} S_{C} \boldsymbol{c}_{\rightarrow}+2 \boldsymbol{c}_{\rightarrow}^{T} S_{C x} \boldsymbol{x}_{\boldsymbol{k}}
$$

siendo $S_{c}$ y $S_{c x}$ las mismas expresiones que las especificadas arriba (en la ecuación (13)).

Como puede observarse en (14), la función de coste es cuadrática respecto de $\boldsymbol{c}_{\rightarrow}$, es decir, respecto de la variable matricial que contiene los grados de libertad del problema de optimización.

Formalización del problema de optimización y determinación de la ley de control correspondiente a la estructura CLP-MPC. De las dos posibilidades que cabría 
considerar, sin restricciones y con restricciones, el caso apropiado en el contexto del presente trabajo es el segundo. Las restricciones más habituales son las que afectan a los valores de las entradas manipulables y/o a sus incrementos, pero también puede haber restricciones en las salidas e incluso en los estados. En cualquier caso, en (Rossiter, 2003) se demuestra que todas las restricciones pueden expresare de modo conjunto mediante una inecuación matricial de la forma genérica siguiente, donde $L$ integra todos los límites o cotas:

$$
M c_{\rightarrow}+N x_{k}-L \leq 0
$$

es decir, que las restricciones pueden formalizarse mediante una expresión afín respecto de las variables $\boldsymbol{c}_{\rightarrow}\left(\right.$ d.o.f) y $\boldsymbol{x}_{k}$ (estado actual), con $L$ (cotas) como término independiente.

Teniendo en cuenta ahora la expresión de la función de coste, mostrada en la ecuación (14) y la de las restricciones, mostrada en la ecuación (15), la formulación del problema de optimización para el caso con restricciones quedará de la forma siguiente:

$$
\begin{gathered}
\min _{\boldsymbol{c}_{\rightarrow}} J_{k(C L P)}=\left(\boldsymbol{c}_{\rightarrow}^{T} S_{c} \boldsymbol{c}_{\rightarrow}+2 \boldsymbol{c}_{\rightarrow}^{T} S_{c x} \boldsymbol{x}_{\boldsymbol{k}}\right) \\
\text { s.t. } \\
M \boldsymbol{c}_{\rightarrow}+N \boldsymbol{x}_{k}-L \leq 0
\end{gathered}
$$

La solución de (16) nos dará la $\boldsymbol{c}_{\rightarrow}$ óptima para el caso con restricciones, $\boldsymbol{c}_{\text {opt } R \rightarrow}$, y el primer elemento de esa secuencia, al que denotaremos como $\boldsymbol{c}_{k R}$, será el término complementario que habrá que sumar a la ley de control base para obtener la acción de control predictivo CLP-MPC, con restricciones, correspondiente al instante $k$, es decir:

$$
\boldsymbol{u}_{k R}=-K \boldsymbol{x}_{k}+\boldsymbol{c}_{k R}
$$

\section{Estrategia de control predictivo FMBPC aplicada al control de fangos activados}

La estrategia FMBPC abarca distintas alternativas, dependiendo de los principios y métodos aplicados para obtener la ley de control. La ley FMBPC tomada como referencia en el presente trabajo se dedujo (como se indicó en la Introducción) aplicando el denominado principio de equivalencia, propio del control PFC, obteniéndose como resultado una ley de control analítica y explícita. La forma original de esta ley fue deducida en (Vallejo et al., 2019), particularizada para el caso de un proceso de depuración de aguas residuales mediante fangos activados. Este tipo de proceso biológico ha sido elegido también como caso de estudio en nuestro trabajo y se describe brevemente a continuación, tanto el propio proceso, como el modelado matemático del mismo.

\subsection{Proceso biológico de depuración de aguas residuales mediante fangos activados: identificación y modelado}

El caso de estudio considerado consiste en un proceso de depuración biológica de aguas residuales mediante fangos activados que se caracteriza principalmente por lo siguiente: tiene una dinámica compleja debido a su carácter biológico, es fuertemente no lineal y es multivariable. A efectos de reducción de la complejidad, para un mejor tratamiento, se ha considerado un modelo simplificado, pero manteniendo en lo esencial las interesantes características de estudio que acabamos de enunciar.

En (Vallejo et al., 2019) puede verse una descripción detallada de esta planta y de su dinámica. A continuación, en la Figura 1, se representa de modo esquemático tal proceso, incluyéndose las principales variables físico-químicas relacionadas. Desde el punto de vista de nuestro sistema de control, esta planta es vista como un sistema multivariable, con tres entradas y dos salidas: una entrada manipulable, dos perturbaciones en la entrada y dos salidas controladas. Esta configuración de entrada-salida es mostrada en la Figura 2, donde se incluye también cuáles son las variables implicadas, su rol en el marco del sistema de control y su significado físico:

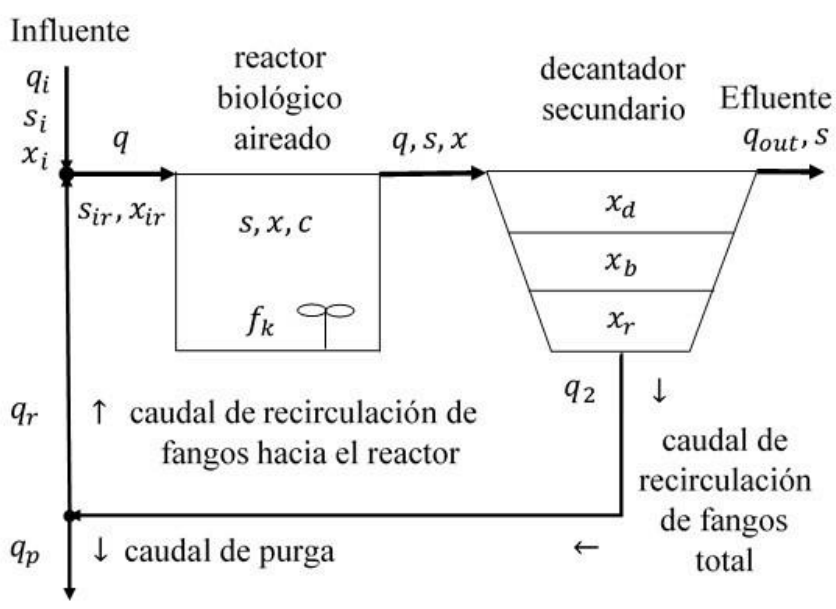

Figura 1: Proceso biológico de fangos activados simplificado.

\section{Perturbaciones}

di (caudal de entrada: $q_{i}\left(\mathrm{~m}^{3} / \mathrm{h}\right)$ )

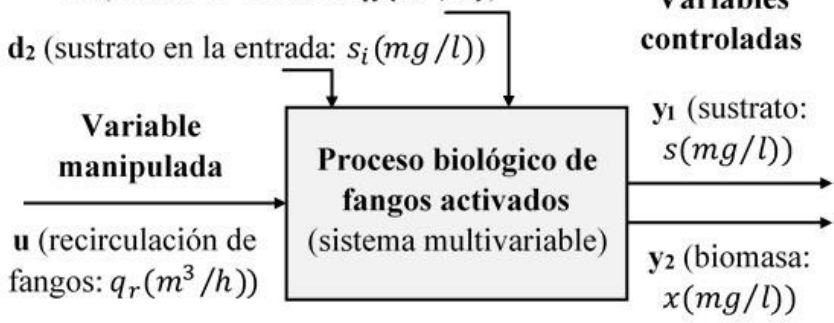

Figura 2: Sistema multivariable: tres entradas y dos salidas.

Modelo matemático fisicoquímico. Nuestro trabajo se ha llevado a cabo en simulación, representando la planta mediante un modelo matemático continuo, constituido por ecuaciones diferenciales que describen los balances de masa del sustrato, la biomasa y el oxígeno (en el reactor y en el decantador). El modelo utilizado puede consultarse en (Francisco et al., 2006; Vallejo et al., 2019) y consiste en una simplificación del modelo standard conocido como Activated Sludge Model No. 1 (ASM1) (Henze et. al. ,1987).

Identificación borrosa. La estrategia FMBPC considerada en este trabajo utiliza como modelo base para para llevar a cabo las predicciones un modelo borroso de la planta, de tipo TS. Este modelo se obtiene mediante identificación borrosa, en nuestro caso a partir de series de datos de entrada-salida de la planta obtenidos mediante simulación en lazo abierto (aunque 
podrían proceder también de experimentos reales). Las series de datos temporales de entrada utilizados, agrupados en matrices de tres columnas (dos para las perturbaciones y una para la variable manipulada), contienen valores típicos de una planta real de tamaño medio que fue tomada como referencia. Esta planta es la depuradora municipal de la ciudad de Manresa (Barcelona, Spain), estudiada en su etapa inicial, en la década de los años 1990. En esas fechas se registraron series de datos reales de entrada-salidas correspondientes a diferentes campañas llevadas a cabo, siendo utilizados esos datos en trabajos de control predictivo de procesos de fangos activados (Moreno, 1994). En el presente trabajo también se han tomado como referencia esas colecciones de datos, utilizando los datos de entrada, o valores cercanos a los mismos, en los experimentos de simulación en lazo abierto. Como resultado de estas simulaciones se obtuvieron los correspondientes datos de salida.

A partir de los datos numéricos de entrada-salida obtenidos en simulación se desarrollaron diversos procesos de identificación borrosa, cada uno de los cuáles produjo como resultado un modelo borroso discreto de la planta en forma de reglas cualitativas del tipo si-entonces, con diferentes estructuras, valores de los parámetros de identificación y grados de validación (véase: Vallejo et al., 2019, 2021). En estos modelos, cada una de las reglas representa un clúster o submodelo, cuya validez será mayor cuanto mayor sea el grado de pertenencia de cada una de las componentes del vector antecedente instantáneo respecto de los correspondientes conjuntos borrosos. El vector antecedente está constituido por un cierto conjunto de variables que, en mayor o menor grado, influyen en la salida descrita de la planta. La identificación fue realizada mediante software desarrollado en el entorno Matlab® \& Simulink® (The MathWorks, Inc., Natick, Massachusetts, USA), haciendo uso de la herramienta de software denominada FMID (Fuzzy Model Identification Toolbox) (Babuška, 1998b), adaptada convenientemente para su correcto funcionamiento en nuestro caso. La herramienta FMID está basada en técnicas de clusterización por medio del algoritmo de Gustafson-Kessel y fue desarrollada como soporte del libro Fuzzy Modelling for Control (Babuška, 1998a).

Modelo equivalente en el espacio de estados. Los modelos del proceso de fangos activados identificados por la herramienta FMID, de tipo borroso y discreto, fueron transformados en (Vallejo et al., 2019) en modelos discretos equivalentes en el espacio de estados, lineales, pero con matrices dependientes del tiempo, es decir, modelos de tipo DLTV. Mostramos a continuación la forma del modelo equivalente:

$$
\begin{gathered}
\boldsymbol{z}_{\boldsymbol{m}}(k+1)=\overline{\boldsymbol{A}}_{\boldsymbol{m}} \boldsymbol{z}_{\boldsymbol{m}}(k)+\overline{\boldsymbol{B}}_{\boldsymbol{m}} \boldsymbol{u}_{\boldsymbol{a}}(k)+\overline{\boldsymbol{R}}_{\boldsymbol{m}} \\
\boldsymbol{y}_{\boldsymbol{m}}(k)=\overline{\boldsymbol{C}}_{\boldsymbol{m}} \boldsymbol{z}_{\boldsymbol{m}}(k)
\end{gathered}
$$

donde:

$\boldsymbol{z}_{\boldsymbol{m}}(k)$ : vector de estado extendido, integrado por las dos salidas y las dos perturbaciones

$\boldsymbol{u}_{\boldsymbol{a}}(k)=\left(\begin{array}{c}u(k) \\ u(k-1)\end{array}\right)$ : vector de entrada extendido, integrado por la variable manipulable en los instantes actual, $u(k)$ y anterior, $u(k-1)$ $\boldsymbol{y}_{\boldsymbol{m}}(k)$ : vector de salida, integrado por las dos salidas controladas

$\overline{\boldsymbol{A}}_{m}(k), \overline{\boldsymbol{B}}_{m}(k), \overline{\boldsymbol{R}}_{m}(k)$ y $\overline{\boldsymbol{C}}_{m}(k)$ : matrices del sistema en el espacio de estados

\subsection{Ley de control FMBPC}

Haciendo uso del modelo que acaba de ser especificado y utilizando el principio de equivalencia (generalizado para sistemas multivariables), en (Vallejo et al., 2019) se dedujo una ley de control predictivo FMBPC, analítica y explícita, para el mismo caso de estudio que estamos considerando, es decir, para el proceso de fangos activados. La ley de control deducida es la siguiente, donde $u(k)$ es la entrada manipulable $q_{r}(k)$ (se omiten, por simplicidad, las expresiones matemáticas correspondientes al resto de variables y coeficientes):

$$
\begin{aligned}
u(k)=\boldsymbol{P}_{\mathbf{1 0}} \boldsymbol{M}_{\boldsymbol{a}}{ }^{-1}\left(\boldsymbol{y}_{\boldsymbol{r}}(k+H)-\boldsymbol{y}(k)+\boldsymbol{y}_{\boldsymbol{m}}(k)\right. & \\
& -\overline{\boldsymbol{C}}_{\boldsymbol{m}} \overline{\boldsymbol{A}}_{\boldsymbol{m}}{ }^{H} \boldsymbol{z}_{\boldsymbol{m}}(k) \\
-\overline{\boldsymbol{C}}_{\boldsymbol{m}}\left(\overline{\boldsymbol{A}}_{\boldsymbol{m}}{ }^{\boldsymbol{H}-\mathbf{1}}+\right. & \left.\left.\left(\overline{\boldsymbol{A}}_{\boldsymbol{m}}^{\boldsymbol{H - 1}}-\boldsymbol{I}\right)\left(\overline{\boldsymbol{A}}_{\boldsymbol{m}}-\boldsymbol{I}\right)^{-\mathbf{1}}\right) \overline{\boldsymbol{R}}_{\boldsymbol{m}}\right)
\end{aligned}
$$

Observando la expresión anterior podemos concluir que $u(k)$ depende de las matrices $\overline{\boldsymbol{A}}_{m}, \overline{\boldsymbol{B}}_{m}, \overline{\boldsymbol{R}}_{m}$ y $\overline{\boldsymbol{C}}_{m}$, es decir, del modelo equivalente DLTV mostrado en la ecuación (18), de las trayectorias de referencia fijadas para las salidas $\left(\boldsymbol{y}_{\boldsymbol{r}}\right)$ y del parámetro de control predictivo $H$ (horizonte de coincidencia).

La ley de control FMBPC mostrada en la ecuación (19) será tomada como referencia en el presente trabajo, pero la expresión utilizada no será exactamente la misma, sino una expresión equivalente, justificada y detallada en la sección siguiente.

\section{Integración de la estrategia FMBPC en una estructura de control predictivo CLP-MPC para el manejo de restricciones}

El principal objetivo del presente trabajo consiste en integrar la estrategia FMBPC en un esquema de control predictivo CLP-MPC, como mecanismo para el manejo de restricciones, poniendo el foco en este caso en las restricciones de la acción de control. En la sección 2 de este artículo se expusieron los fundamentos del control predictivo en modo dual, en general, y para la estructura CLP-MPC en particular. La hipótesis de partida de los desarrollos mostrados es la existencia de un modelo lineal en el espacio de estados, representativo de la planta objeto de control, que pueda ser utilizado como modelo de predicciones. Así mismo, será necesaria una ley de control base, estabilizante, habitualmente una ley de realimentación de estados, que será utilizada en todo el horizonte de predicción de la forma siguiente: en el modo1, acompañada de un término aditivo sujeto a optimización y en el modo 2, sin ese término (o considerando que es nulo siempre). Es necesario, por tanto, elegir para nuestro caso ambas componentes de la estructura CLP-MPC.

\subsection{Modelo de predicciones y ley de control base}

Modelo de predicciones. El modelo del proceso de fangos activados detallado en la ecuación (18) fue reformulado en (Vallejo et al., 2021), obteniéndose otro modelo equivalente 
(también de tipo DLTV) y a partir de él, tomando como referencia un punto de equilibrio de la planta en lazo abierto, previamente elegido, se dedujo un modelo incremental local para el mismo proceso. El modelo deducido es un modelo en el espacio de estados, de tipo DLTI, válido para estados próximos al estado estacionario de referencia y tiene la forma siguiente:

$$
\begin{gathered}
\boldsymbol{x}_{\boldsymbol{i n c}}(k+1)=\overline{\boldsymbol{A}}_{\boldsymbol{m} \boldsymbol{N}} \boldsymbol{x}_{\boldsymbol{i n c}}(k)+\overline{\boldsymbol{B}}_{\boldsymbol{m} \boldsymbol{N}} \boldsymbol{u}_{\boldsymbol{i n c}}(k) \\
\boldsymbol{y}_{\boldsymbol{i n c}}(k)=\overline{\boldsymbol{C}}_{\boldsymbol{m} \boldsymbol{N}} \boldsymbol{x}_{\boldsymbol{i n c}}(k)
\end{gathered}
$$

donde:

$$
\begin{gathered}
\overline{\boldsymbol{A}}_{\boldsymbol{m} \boldsymbol{N}}, \quad \overline{\boldsymbol{B}}_{\boldsymbol{m} \boldsymbol{N}} \text { y } \overline{\boldsymbol{C}}_{\boldsymbol{m} \boldsymbol{N}} \text { son las matrices } \\
\text { correspondientes al estado estacionario } \\
\boldsymbol{x}_{\boldsymbol{i n c}}(k+1), \quad \boldsymbol{x}_{\boldsymbol{i n c}}(k), \quad \boldsymbol{u}_{\boldsymbol{i n c}}(k), \boldsymbol{y}_{\boldsymbol{i n c}}(k): \\
\text { variables incrementales con respecto al } \\
\text { punto de equilibrio }
\end{gathered}
$$

El modelo DLTI mostrado en la ecuación (20) es el que se utilizará como modelo de predicciones para representar al proceso de fangos activados en la estructura CLP-MPC. En la implementación online de la estrategia mixta FMBPC/CLP, para cada instante de muestreo $k$ habrá un modelo diferente, dependiente de $k$, pero el modelo que se transferirá a la estructura CLP-MPC se mantendrá constante en ella durante las predicciones.

Ley de control base FMBPC. Partiendo de la expresión original de la ley de control FMBPC (ecuación (19)) y teniendo en cuenta la relación entre los diferentes modelos DLTV deducidos en (Vallejo et al., 2019, 2021) a los que hemos hecho referencia anteriormente, en (Vallejo et al., 2021) se demuestra que la ley de control FMBPC es equivalente, para el modelo incremental local mostrado en (20), a la expresión siguiente:

$$
\boldsymbol{u}_{\text {inc }}(k)=-\left(\boldsymbol{M}_{\boldsymbol{a N}}{ }^{-1} \overline{\boldsymbol{C}}_{\boldsymbol{m} \boldsymbol{N}} \overline{\boldsymbol{A}}_{\boldsymbol{m} \boldsymbol{N}}{ }^{\boldsymbol{H}}\right) \boldsymbol{x}_{\boldsymbol{i n c}}(k)
$$

donde $\boldsymbol{M}_{\boldsymbol{a} \boldsymbol{N}}$ es una expresión matricial que se obtiene a partir de $\boldsymbol{M}_{\boldsymbol{a}}$ (matriz implícita en la ecuación (19)).

La ley de control obtenida es compatible con la conocida ley de realimentación de estados $\boldsymbol{u}=-\boldsymbol{K} \boldsymbol{x}$, es decir, la expresión mostrada en la ecuación (21) puede ser formalizada de manera análoga, como vemos a continuación:

$$
\begin{array}{r}
\boldsymbol{u}_{\boldsymbol{i n c}}(k)=-\boldsymbol{K} \boldsymbol{x}_{\boldsymbol{i n c}}(k) \\
\text { donde: } \quad \boldsymbol{K}=\left(\boldsymbol{M}_{\boldsymbol{a} \boldsymbol{N}}{ }^{-1} \overline{\boldsymbol{C}}_{\boldsymbol{m} \boldsymbol{N}} \overline{\boldsymbol{A}}_{\boldsymbol{m} \boldsymbol{N}}{ }^{\boldsymbol{H}}\right)
\end{array}
$$

En la implementación online de la estrategia mixta FMBPC/CLP, para cada instante de muestreo $k$ habrá una $\boldsymbol{K}$ diferente, dependiente de $k$, pero esa $\boldsymbol{K}$ se mantendrá constante en el cálculo de predicciones de la estructura CLP-MPC correspondiente a ese instante de muestreo.

En (Vallejo et al., 2021) se demuestra que la ley de control mostrada en (21) y (22) garantiza la estabilidad (local) del sistema de control en lazo cerrado y, por tanto, podemos adoptarla para la estructura CLP-MPC como la ley de control base estabilizante, en los cálculos de las predicciones.

En la siguiente sección se utilizará una notación alternativa para las variables implicadas en esta ley de control, incorporando, por un lado, información relativa a su origen, denotando: $\boldsymbol{u}_{\text {inc }}(k) \equiv u_{\text {inc.pred } \mid F M B P C}$, y simplificando, por otro lado, la notación referente al estado: $\boldsymbol{x}_{\boldsymbol{i n c}}(k) \equiv \boldsymbol{x}(k)$. Con esos cambios, la ecuación (22) queda así:

$$
u_{\text {inc.pred } \mid F M B P C}=-\boldsymbol{K} \boldsymbol{x}(k)
$$

\subsection{Implementación de la estrategia mixta FMBPC/CLP}

Tomando el modelo (incremental) mostrado en (20) como modelo de predicciones y la ley FMBPC mostrada en (23) como ley de control base del esquema de predicciones CLP-MPC, podremos hacer uso de las expresiones matemáticas mostradas en la sección 2 para una estructura genérica CLP-MPC. Así, el problema de imposición de restricciones a la ley de control FMBPC será equivalente a resolver un problema de optimización como el expresado en la ecuación (16) (con las particularidades matemáticas necesarias para adaptarlo a nuestro caso de estudio), cuya solución, en cada instante $k$, consistirá en una secuencia óptima de perturbaciones de la ley de control a lo largo del horizonte de predicción, $\boldsymbol{c}_{o p t R \rightarrow}$. El primer elemento de esa secuencia, $\boldsymbol{c}_{k R}$, será la perturbación óptima que deberá sumarse online a la ley de control base, obteniendo la acción de control óptima que garantizará el cumplimiento de las restricciones: $\boldsymbol{u}_{k R}=-K \boldsymbol{x}_{k}+\boldsymbol{c}_{k R}$ (ecuación (17)). Por otra parte, dado que el modelo usado en la estructura CLP-MPC es un modelo incremental, a continuación habrá que sumarle a $\boldsymbol{u}_{k R}$ la acción de control correspondiente al estado estacionario, que denotaremos con $u_{s s}$. En la implementación online de la estrategia mixta FMBPC/CLP este proceso se repetirá sistemáticamente cada período de muestreo, obteniendo en cada iteración un valor óptimo de la acción de control, dependiente de $k$. Este procedimiento puede formalizarse matemáticamente mediante la siguiente secuencia de expresiones (ecuaciones (24) a (27)):

donde:

$$
u_{\text {opt }}(k)=u_{\text {inc.pred } \mid F M B P C}+c_{\text {opt }}(k)
$$

$u_{\text {opt }}(k)$ : acción de control óptima en $k$ (correspondiente al modelo incremental de predicciones), calculada mediante la estructura CLP-MPC (en las Figuras 4c, 4d, 4e y 5c, 5d y 5e: $\left.u_{\text {opt }}(k) \equiv u_{\text {mod.pred }}(k)\right)$

$u_{\text {inc.pred } \mid F M B P C}$ : ley de control base

$c_{\text {opt }}(k)$ : perturbación óptima de la acción de control en $k$, calculada mediante la estructura CLP-MPC (en las Figuras 4c, 4d, 4e y 5c, 5d y 5e: $\left.c_{\text {opt }}(k) \equiv c(k)\right)$

que puede expresarse también de la forma siguiente (utilizando la ecuación (23)):

$$
u_{\text {opt }}(k)=-\boldsymbol{K} \boldsymbol{x}(k)+c_{\text {opt }}(k)
$$

donde:

$$
\begin{aligned}
& -\boldsymbol{K} \boldsymbol{x}(k) \text { : ley de control base FMPPC } \\
& \boldsymbol{x}(k) \text { : vector de estado correspondiente al modelo } \\
& \text { incremental de predicciones }
\end{aligned}
$$


y sumándole a $u_{o p t}(k)$, expresado según la ecuación (24), la acción de control correspondiente al estado estacionario de referencia, $u_{s s}$ y reordenando, obtendremos la acción de control global, $u_{F M B P C / C L P}(k)$ :

$$
\begin{aligned}
u_{F M B P C} / C L P & (k)=u_{o p t}(k)+u_{s S} \\
& =\left(u_{\text {inc.pred } \mid F M B P C}+c_{o p t}(k)\right)+u_{s s} \\
& =\left(u_{\text {inc.pred } \mid F M B P C}+u_{s S}\right)+c_{o p t}(k)
\end{aligned}
$$

que puede ser expresada finalmente, de forma más sencilla y compacta, como sigue:

$$
u_{F M B P C / C L P}(k)=u_{\text {pred } \mid F M B P C}+c_{o p t}(k)
$$

donde: $\quad u_{\text {pred } \mid F M B P C}=\left(u_{\text {inc.pred } \mid F M B P C}+u_{s S}\right)$

La ecuación (27) representa la expresión final de la implementación online, en cada instante $k$, de la ley de control correspondiente a la estrategia mixta FMBPC/CLP. En la práctica es equivalente a calcular la acción de control predictiva FMBPC con el modelo global y sumarle $c_{\text {opt }}(k)$, si asumimos que, para estados cercanos al estado estacionario de referencia, el modelo global es equivalente al modelo local incremental. La ley de control FMBPC/CLP obtenida está optimizada para el cumplimiento de las restricciones, que en nuestro caso de estudio se han concretado en las variaciones de la acción de control, en forma de cotas, máximas y mínimas. Por último, en relación con la estabilidad de la estrategia, asumiremos la conclusión establecida al respecto en (Rossiter, 2003) respecto a la estructura CLP-MPC, que establece que si la ley de control base en lazo cerrado es estabilizante, la estrategia de control predictivo en lazo cerrado será estable.

El esquema de implementación online de la estrategia FMBPC/CLP propuesta en el presente artículo se muestra en la Figura 3 y mediante el Algoritmo 1 se describe y explica de manera ordenada el procedimiento de implementación seguido. Para cada instante $k$ se calcula, por un lado la acción de control predictivo $u_{\text {pred } \mid F M B P C}(k)$, y por otro, haciendo uso de la estructura CLP-MPC, la perturbación de control óptima $c_{\text {opt }}(k)$ que garantiza la satisfacción de las restricciones. Este segundo cálculo se realiza mediante la estructura CLP-MPC, a partir de la información transmitida por la estructura FMBPC, relativa a las matrices del modelo de predicciones, $\overline{\boldsymbol{A}}_{\boldsymbol{m} N}(k)$, $\overline{\boldsymbol{B}}_{\boldsymbol{m} \boldsymbol{N}}(k), \overline{\boldsymbol{C}}_{\boldsymbol{m} \boldsymbol{N}}(k)$ y al factor $\boldsymbol{K}(k)$ de la ley de control base, todo ello actualizado para el instante de muestreo actual, $k$. El cálculo de predicciones y el procedimiento de optimización son realizados dentro de la estructura CLP-MPC utilizando un modelo lineal, pero el procedimiento en su conjunto es no lineal, dado que en el transcurso del tiempo de implementación, en cada iteración, se actualizan $\overline{\boldsymbol{A}}_{\boldsymbol{m} \boldsymbol{N}}, \bar{B}_{\boldsymbol{m} \boldsymbol{N}}$, $\overline{\boldsymbol{C}}_{\boldsymbol{m} \boldsymbol{N}}$ y $\boldsymbol{K}$. Tenemos por tanto que la estrategia mixta de control predictivo descrita es, globalmente, no lineal.

\section{Algoritmo 1. Implementación de la estrategia FMBPC/CLP}

1. Elección del modelo borroso FM (identificado a partir de series de datos de entrada/salida).
2. Elección de patrones para las perturbaciones de entrada (conforme a series de datos experimentales).

3. Elección de referencias y trayectorias de referencia.

4. Inicialización de variables: punto de inicio, vector de estado, vector antecedente.

5. Elección de parámetros de la estrategia de control FMBPC y de la estructura CLP. Principales parámetros: horizonte de coincidencia/FMBPC $(H)$; número de pasos del modo 1 de la estructura CLP $\left(n_{c}\right)$; cotas superior e inferior para la variable manipulada del modelo de predicciones de la estructura CLP $\left(u_{\text {máx }}\right.$ y $\left.u_{\text {mín }}\right)$.

6. Actualización de los modelos DLTV/DLTI en el instante de tiempo actual, $k$.

7. Cálculo/actualización de la $\boldsymbol{K}(k)$ correspondiente a la ley de control base $(-\boldsymbol{K} \boldsymbol{x})$.

8. Cálculo de la variable de control FMBPC correspondiente al instante de tiempo actual: $u_{\text {pred }}(k)$.

9. Envío a la estructura CLP de las matrices del modelo de predicciones, $\overline{\boldsymbol{A}}_{\boldsymbol{m} \boldsymbol{N}}(k), \overline{\boldsymbol{B}}_{\boldsymbol{m} \boldsymbol{N}}(k)$ y $\overline{\boldsymbol{C}}_{\boldsymbol{m} \boldsymbol{N}}(k)$ y del factor $\boldsymbol{K}(k)$ de la ley de control base.

10. Cálculo de la perturbación de la acción de control, $c(k)$, mediante optimización dentro de la estructura CLP.

11. Cálculo de la variable de control de la estrategia mixta FMBPC/CLP en $k: u_{F M B P C / C L P}(k)=u_{\text {pred }}(k)+c(k)$. Aplicación de la variable de control $u_{F M B P C / C L P}(k) \equiv$ $u(k)$ al proceso de fangos activados.

12. Si $t<t_{\text {simul }}$, volver al paso 6; si $t=t_{\text {simul }}$, terminar.

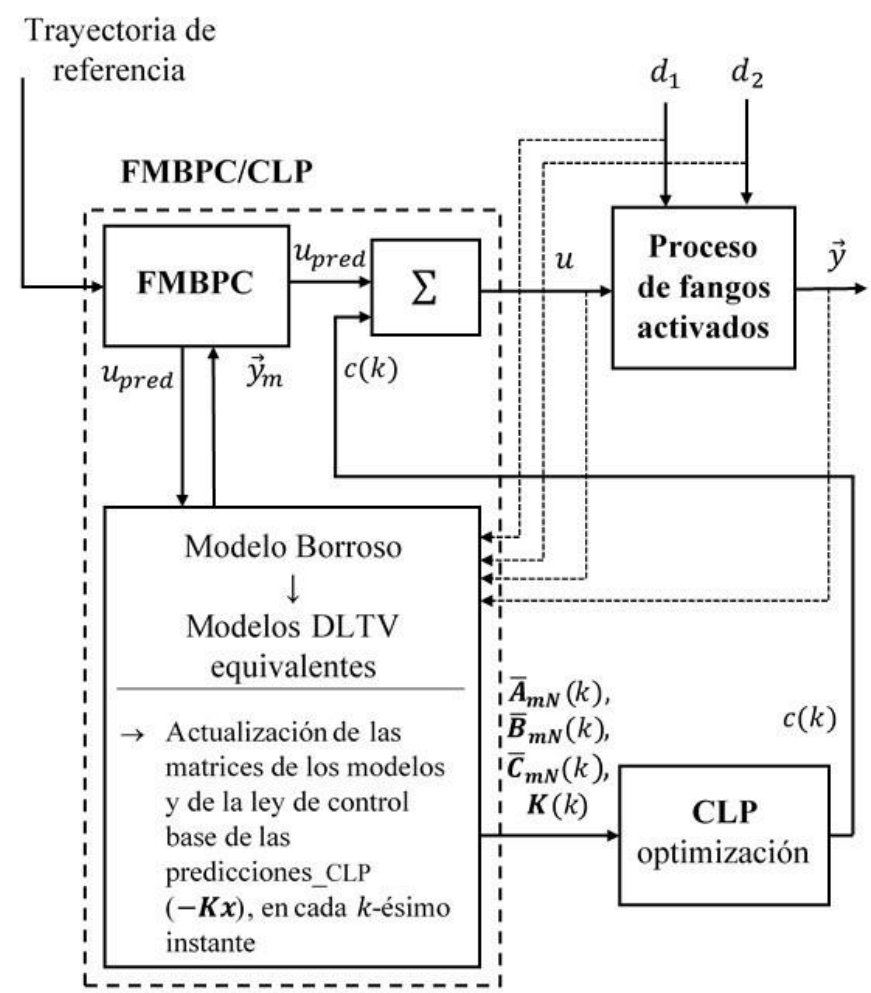

Figura 3. Estrategia mixta FMBPC/CLP: esquema de implementación. 


\section{Experimentos: simulación y resultados}

La estrategia FMBPC/CLP propuesta fue aplicada en simulación al control multivariable de un proceso de fangos activados. La implementación se llevó a cabo utilizando el mismo entorno de software que el utilizado en la identificación, es decir, el entorno Matlab® \& Simulink®. Las variables objeto de control simultáneo en los experimentos realizados fueron dos: la concentración de sustrato en el efluente y la concentración de biomasa en el reactor. Desde el punto de vista medioambiental, la principal variable a controlar es la concentración de sustrato, pero el control de la biomasa también tiene su importancia desde el punto de vista operativo en las plantas de tratamiento de aguas residuales. Además, el control simultáneo de esas dos variables es planteado como caso de estudio de una estrategia de control predictivo borroso multivariable. Por tanto, nos interesa por igual el control de ambas variables. En los experimentos realizados, la referencia de la concentración de sustrato fue mantenida en un valor constante a lo largo del tiempo, un valor bajo, supuestamente compatible con el máximo previsto por la legislación medioambiental, mientras que la referencia de la concentración de biomasa fue variable (constante por tramos, pero con dos saltos en dos momentos diferentes del tiempo).

Se muestra a continuación, en la Tabla 1, la configuración de dos de los muchos experimentos realizados, a los que hemos denominado caso 1 y caso 2 , respectivamente, caracterizados por tener dos secuencias diferentes para la referencia de la concentración de biomasa y, en consecuencia, dos zonas de operación diferentes. Las referencias de la concentración de sustrato se mantuvieron constantes en el tiempo y con el mismo valor en ambos casos. Para cada uno de los dos casos se consideraron tres subcasos, correspondientes a tres pares de valores diferentes de las cotas mínima y máxima de la acción de control del modelo local de predicciones. El punto de inicio de los experimentos realizados $\left(s_{0}, x_{0}\right)$, fue el mismo en ambos casos: $s_{0}=200(\mathrm{mg} / \mathrm{l})$ y $x_{0}=2000(\mathrm{mg} / \mathrm{l})$. En la Tabla 1 se detallan los valores o referencias de los diferentes parámetros, tanto de la estrategia FMBPC, como de la estructura CLP-MPC, así como los parámetros de simulación. En relación con la estrategia FMBPC cabe resaltar la importancia del modelo borroso de predicciones $(F M)$. Sin embargo, los detalles del modelo elegido $\left(F M_{1}\right)$ no pueden ser incluidos en la Tabla, debido a las limitaciones de espacio del artículo. Pueden verse sus características en (Vallejo et al., 2021). Y en relación con los parámetros de sintonía $Q$ y $R$, en los experimentos correspondientes a los resultados mostrados se eligió un valor unitario para $R$, es decir $R=1$, y para $Q$ se consideró la relación matemática $Q=C^{\prime} C$, obteniéndose en nuestro caso: $Q=C^{\prime} C=\left(\begin{array}{ll}1 & 0 \\ 0 & 1\end{array}\right)\left(\begin{array}{ll}1 & 0 \\ 0 & 1\end{array}\right)=\left(\begin{array}{ll}1 & 0 \\ 0 & 1\end{array}\right)$, lo cual puede ser expresado formalmente también como sigue: $Q=$ $\left(\begin{array}{cc}Q_{1} & 0 \\ 0 & Q_{2}\end{array}\right)$, con $Q_{1}=1$ y $Q_{2}=1$ (se probaron algunas otras parametrizaciones, pero sin variaciones significativas).

Tabla 1: Configuración de los experimentos de control realizados utilizando la estrategia mixta FMBPC/CLP

\begin{tabular}{|c|c|c|c|c|c|c|c|c|c|c|c|c|c|c|c|c|c|}
\hline \multirow{2}{*}{\multicolumn{2}{|c|}{ Caso }} & \multirow[t]{2}{*}{$\mathrm{VC}$} & \multirow[t]{2}{*}{ Pert. } & \multicolumn{4}{|c|}{ Estrategia FMBPC } & \multicolumn{6}{|c|}{ Estructura CLP-MPC } & \multicolumn{4}{|c|}{ Parámetros de simulación } \\
\hline & & & & $F M$ & $a_{r_{1}}$ & $a_{r_{2}}$ & $H$ & $Q_{1}$ & $Q_{2}$ & $R$ & $n_{c}$ & $u_{\min }$ & $u_{\text {máx }}$ & I. sim. & $s_{\text {ref }}$ & $x_{\text {ref }}($ saltos $)$ & t. salto \\
\hline \multirow{3}{*}{1} & $1 a$ & \multirow{3}{*}{$s, x$} & \multirow{3}{*}{$D_{A}$} & \multirow{3}{*}{$F M_{1}$} & \multirow{3}{*}{0.76} & \multirow{3}{*}{0.96} & \multirow{3}{*}{6} & \multirow{3}{*}{1} & \multirow{3}{*}{1} & \multirow{3}{*}{1} & \multirow{3}{*}{42} & -200 & 200 & \multirow{3}{*}{0 a 166} & \multirow{3}{*}{55} & \multirow{3}{*}{$\begin{array}{l}1800 \text { a } 2200 \\
2200 \text { a } 2000\end{array}$} & \multirow{3}{*}{$\begin{array}{l}31 \\
81\end{array}$} \\
\hline & $1 b$ & & & & & & & & & & & -100 & 100 & & & & \\
\hline & $1 c$ & & & & & & & & & & & -5 & 5 & & & & \\
\hline \multirow{3}{*}{2} & $2 a$ & \multirow{3}{*}{$s, x$} & \multirow{3}{*}{$D_{A}$} & \multirow{3}{*}{$F M_{1}$} & \multirow{3}{*}{0.76} & \multirow{3}{*}{0.96} & \multirow{3}{*}{6} & \multirow{3}{*}{1} & \multirow{3}{*}{1} & \multirow{3}{*}{1} & \multirow{3}{*}{42} & -200 & 200 & \multirow{3}{*}{0 a 166} & \multirow{3}{*}{55} & & \multirow{3}{*}{$\begin{array}{l}31 \\
81\end{array}$} \\
\hline & $2 b$ & & & & & & & & & & & -100 & 100 & & & 2000 a 1900 & \\
\hline & $2 c$ & & & & & & & & & & & -5 & 5 & & & 1900 a 2000 & \\
\hline
\end{tabular}

donde:

VC: variables controladas $(s, x)$; punto de inicio de los experimentos: $s_{0}=200(\mathrm{mg} / \mathrm{l}), x_{0}=2000(\mathrm{mg} / \mathrm{l})$

Pert.: perturbaciones de entrada $\left(q_{i}\left(\mathrm{~m}^{3} / \mathrm{h}\right)\right.$ y $\left.s_{i}(\mathrm{mg} / \mathrm{l})\right)$; patrón elegido: $D_{A}$ (véase: Vallejo et al., 2021)

$F M$ : modelo borroso (fuzzy model); $F M_{1}$ : FM elegido (véase: Vallejo et al., 2021)

$a_{r_{1}}, a_{r_{2}}$ : parámetros de la trayectoria de referencia (véase: Vallejo et al., 2021)

$H$ : horizonte de coincidencia

$Q_{1}, Q_{2}, R$ : parámetros de sintonía correspondientes a la función de coste $J_{k(C L P)}$ de la estructura CLP-MPC

$n_{c}$ : número de pasos del modo 1 en la estructura CLP

$u_{m i ́ n}, u_{m a ́ x}$ : cotas mínima y máxima, respectivamente, de la acción de control $\left(q_{r}\left(m^{3} / h\right)\right)$ del modelo de predicciones

I. sim. (h. ): intervalo de simulación, en horas

$s_{\text {ref }}(\mathrm{mg} / \mathrm{l})$ : referencia de la concentración de sustrato en el efluente

$x_{\text {ref }}(m g / l)$ : referencia de la concentración de biomasa en el reactor, con dos saltos en dos instantes de tiempo diferentes

t. salto: tiempos de ocurrencia de los saltos en la referencia de la concentración de biomasa, en horas

Para cada uno de los dos casos presentados se hicieron tres pruebas (subcasos) considerando tres parejas de valores diferentes para las cotas $u_{\text {mín }}$ y $u_{\text {máx }}$ de la variable manipulable del modelo de predicciones (las mismas parejas de valores para ambos casos). Los resultados de las pruebas muestran que las secuencias de valores de las perturbaciones 
$c(k)$ obtenidas (calculadas como óptimas para asegurar las restricciones) dependen del caso y, para cada caso, dependen de los valores de las cotas. En lo que se refiere a la acción de control final de la estrategia mixta FMBPC/CLP, $u_{F M B P C / C L P}(k)$, se observó que la secuencia de acciones dependía sobre todo del caso, aunque para cada subcaso presentase algunos matices. Por ello y para no alargar excesivamente el artículo, sólo se mostrará, para cada caso, la $u_{F M B P C / C L P}(k)$ correspondiente a una de las tres pruebas (en el análisis posterior se justificará la elección del subcaso). Los resultados obtenidos, expresados gráficamente, se han agrupado en la Figura 4 (caso 1) y en la Figura 5 (caso 2):

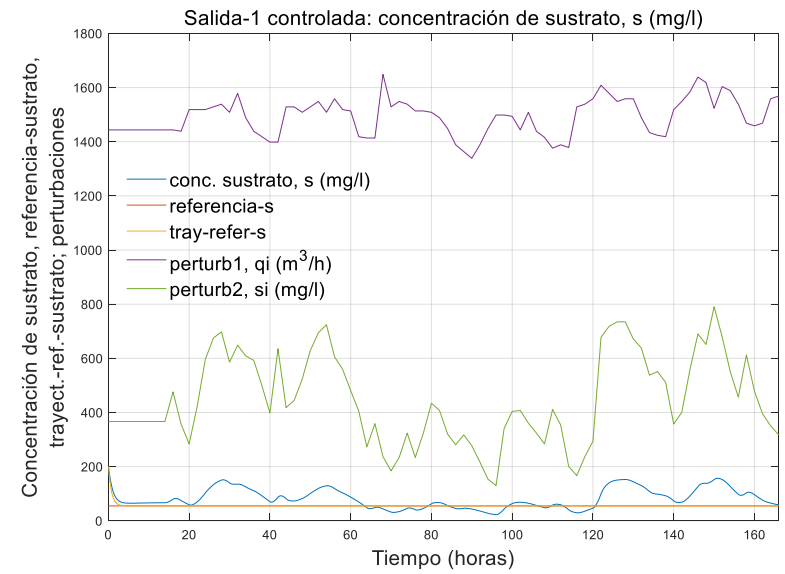

(a) Concentración de sustrato en el efluente y perturbaciones.

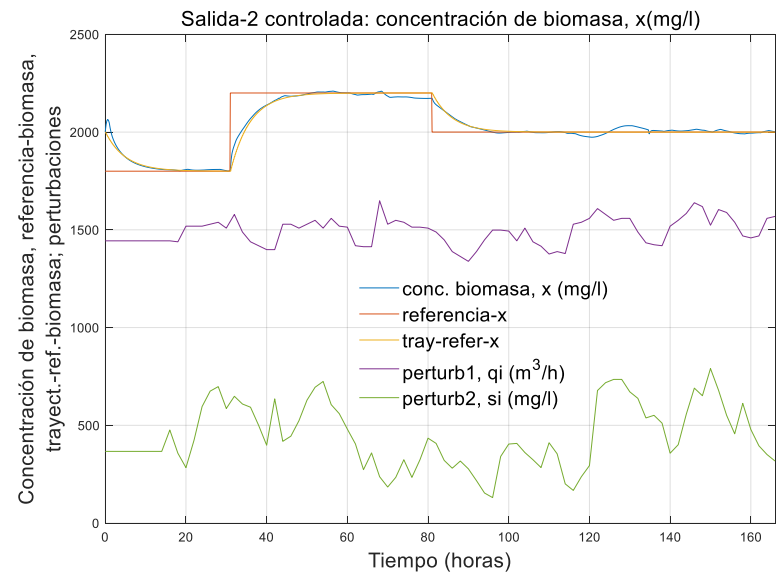

(b) Concentración de biomasa en el reactor y perturbaciones.
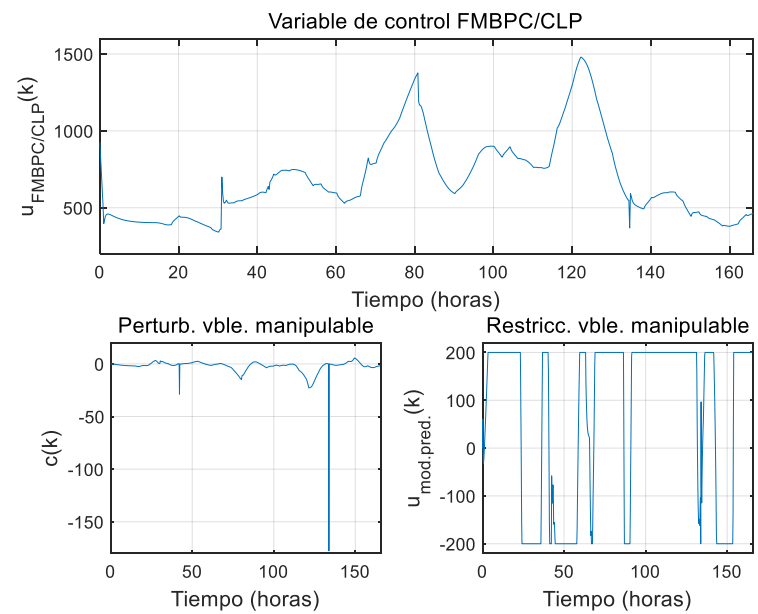

(c) Variable de control global $u_{\mathrm{FMBPC} / \mathrm{CLP}}(k)$, perturbaciones $c(k)$ y restricciones en $u_{\text {mod.pred }}(k)$ (mod. incremental) entre -200 y 200 (caso $\left.1 \mathrm{a}\right)$.
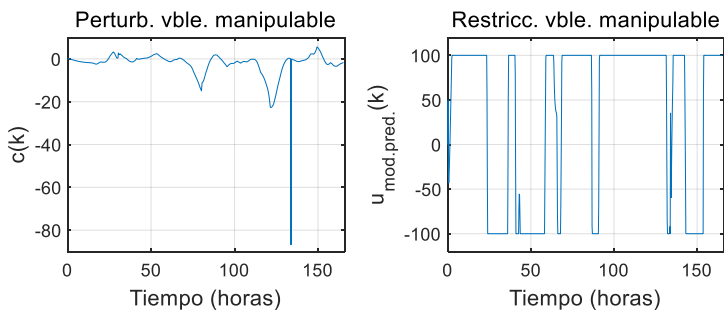

(d) Perturbaciones $c(k)$ y restricciones en $u_{\text {mod.pred }}$

(k) (mod. incremental) entre -100 y 100 (caso $1 \mathrm{~b})$.
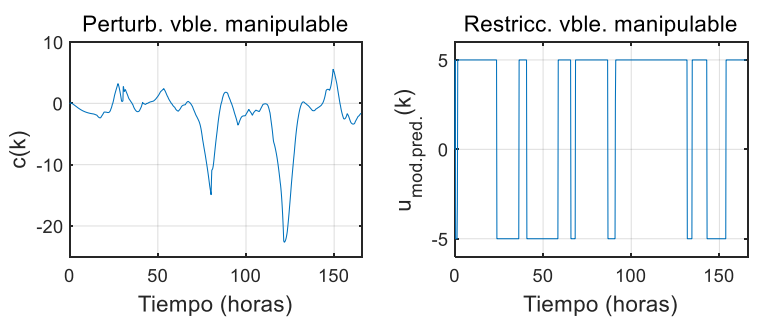

(e) Perturbaciones $c(k)$ y restricciones en $u_{\text {mod.pred }}(k)$ (mod. incremental) entre -5 y 5 (caso 1c).

Figura 4. Experimentos FMBPC/CLP—Caso 1 (1a, 1b y 1c). 


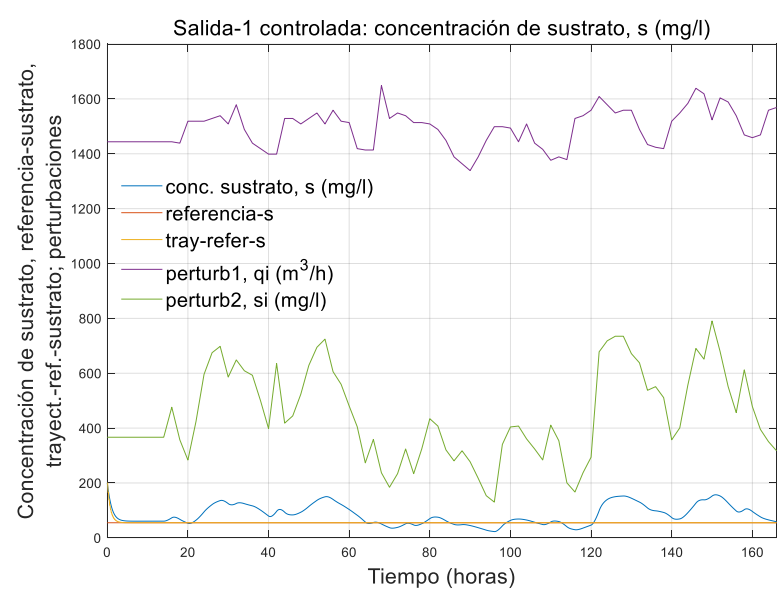

(a) Concentración de sustrato en el efluente y perturbaciones.

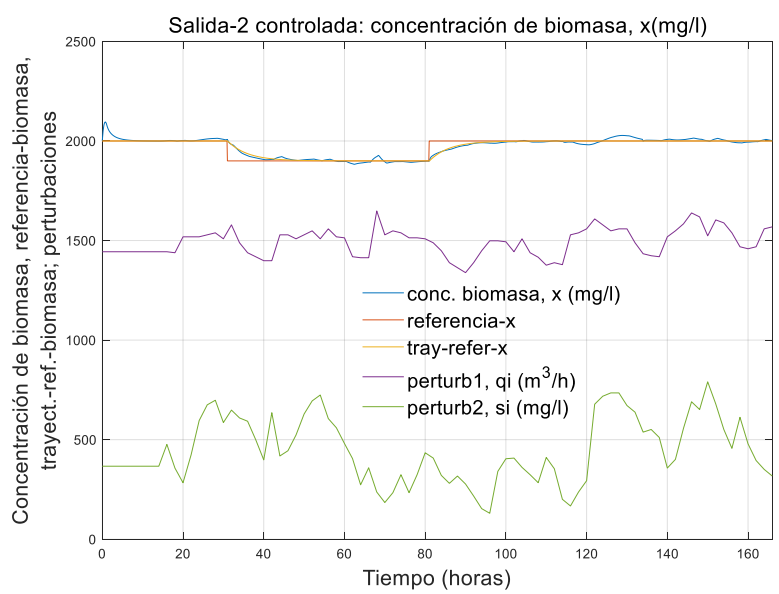

(b) Concentración de biomasa en el reactor y perturbaciones.
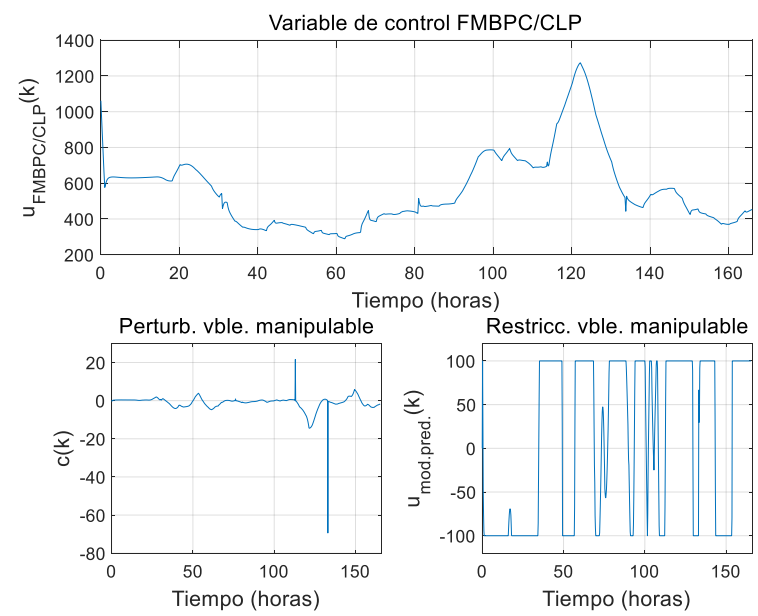

(c) Variable de control global $u_{\mathrm{FMBPC} / \mathrm{CLP}}(k)$, perturbaciones $c(k)$ y restricciones en $u_{\text {mod.pred }}(k)$ (mod. incremental) entre -100 y 100 (caso $2 \mathrm{~b}$ ).
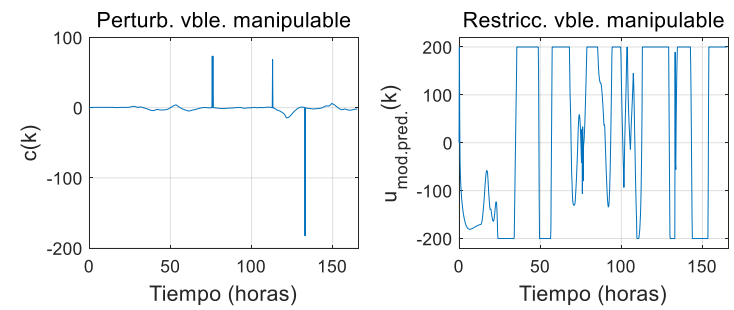

(d) Perturbaciones $c(k)$ y restricciones en $u_{\text {mod.pred }}$

(k) (mod. incremental) entre -200 y 200 (caso 2a).
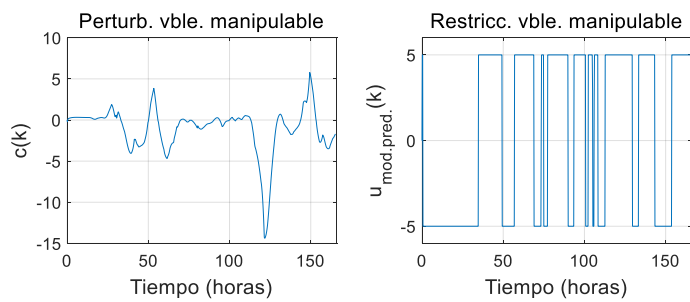

(e) Perturbaciones $c(k)$ y restricciones en $u_{\text {mod.pred }}(k)$ (mod. incremental) entre -5 y 5 (caso 2c).

Figura 5. Experimentos FMBPC/CLP-Caso 2 (2b, 2a y 2c).

Análisis de los resultados. La primera consideración que procede hacer es que el mecanismo propuesto para la imposición de restricciones en la acción de control FMBPC, integrando esta estrategia en una estructura CLP-MPC, es realizable y eficaz. En los 6 subcasos presentados vemos que la perturbación óptima $c(k)$ calculada mediante este mecanismo actúa adecuadamente para que las acciones de control del modelo de predicciones satisfagan las restricciones. Por otra parte, se observa que las secuencias de valores $c(k)$ son diferentes para cada caso y subcaso, como cabría esperar. Así mismo, se observa claramente también que cuanto más grande sea el intervalo de restricciones de las acciones de 
control, menos se saturará en los extremos la acción de control. Este grado de saturación resulta lógico en el contexto de los experimentos considerados, puesto que las perturbaciones de entrada $\left(q_{i}\right.$ y $\left.s_{i}\right)$ son grandes en magnitud y con grandes oscilaciones, de tal forma que el algoritmo de control principal, es decir, el correspondiente a la estrategia FMBPC, trabaja para que las salidas sigan sus trayectorias de referencia a pesar de las perturbaciones, es decir, trata de rechazar las amplias perturbaciones incrementando la acción de control todo lo que sea necesario. Pero claro, cuando la orden del algoritmo tienda a incrementar la acción de control por encima de las cotas, la acción de control se saturará como consecuencia de la actuación del mecanismo de imposición de restricciones implementado. Y esta saturación será menor cuanto mayor sea el margen de actuación del algoritmo principal. Sin embargo, un margen muy amplio para las variaciones de la acción de control puede tener como consecuencia una acción global con cambios bruscos, es decir, no gradual. Estas observaciones nos conducen al razonamiento de que, desde el punto de vista operativo, parece conveniente buscar un equilibrio o compromiso entre las posibles ventajas derivadas de la imposición de un intervalo pequeño para las acciones de control (menor consumo energético, menor esfuerzo de los actuadores o acción de control global más suave o gradual) y las asociadas a una mayor grado de libertad de las acciones de control (capacidad para alcanzar el seguimiento de las trayectorias de referencia en presencia de fuertes perturbaciones).

Por otra parte, además del rechazo de las perturbaciones, otro objetivo habitual de cualquier sistema de control es, naturalmente, el seguimiento de la referencia. En nuestro caso, este objetivo resulta implícito de forma evidente en el propio diseño de los experimentos, los cuales incluyen dos saltos en la referencia de la concentración de la biomasa, diferentes para cada uno de los dos casos. La magnitud de los saltos del caso 2 es menor que la de los saltos del caso 1 y por tanto cabría esperar para el caso 2 una pequeña reducción de la necesidad de variabilidad de la acción de control para responder adecuadamente a esos saltos, lo cual parece corroborarse observando que mientras que en el caso 1 el intervalo de restricciones necesario para que comience a reducirse el grado de saturación de la acción de control es el intervalo $(-200,200)$, en el caso 2 es posible tener una reducción similar de la saturación para restricciones de la acción de control en el intervalo $(-100,100)$.

Por último, en relación con la acción de control final de la estrategia mixta FMBPC/CLP, $u_{F M B P C / C L P}(k)$, a partir de la observación de los resultados correspondientes a ambos casos, cabe deducir dos cosas. Por un lado, que la acción de control final de la estrategia propuesta es capaz de conducir a las dos salidas a sus valores de referencia, simultáneamente, con un seguimiento muy aceptable de las correspondientes trayectorias, para distintas zonas de operación, en presencia de fuertes perturbaciones en la entrada y de forma gradual y sin grandes esfuerzos. Por otro lado, cabe también comentar que, para cada uno de los dos casos, la acción de control final de los distintos subcasos es similar, aunque lógicamente presenta algunos matices derivados del margen de actuación permitido a la acción de control (mediante las restricciones). Solo se ha mostrado la acción de control final de uno de los subcasos (para abreviar la longitud del artículo), siendo el subcaso elegido el primero que permite una cierta reducción de la saturación, según vamos ampliado el margen de actuación.

\section{Conclusiones}

La principal conclusión que podemos extraer del trabajo desarrollado es que se ha conseguido integrar una estrategia de control predictivo borroso de tipo FMBPC, basado en control $\mathrm{PFC}$, en una estructura de control predictivo en modo dual de tipo CLP-MPC, con el objetivo de dotar a la primera de un mecanismo de imposición de restricciones en la acción de control. Este objetivo ha sido alcanzado tomando la ley de control FMBPC analítica y explícita como ley de control base del modo 1 de la estructura CLP-MPC. Los experimentos con nuestro caso de estudio llevados a cabo en simulación corroboran que el mecanismo propuesto es posible y válido. Cabe resaltar la dificultad de la integración de ambas metodologías y la escasez de trabajos relativos a la estructura CLP-MPC.

Otra conclusión importante es que también es posible y útil diseñar técnicas de control avanzado basadas en la confluencia de técnicas de control inteligente con metodologías de control predictivo, en este caso dos metodologías diferentes y con restricciones. La parte inteligente de la estrategia mixta propuesta reside en el tipo de modelo utilizado como base de la estrategia FMBPC, que es un modelo borroso de tipo TS. La utilización de modelos borrosos, es decir de modelos cualitativos, obtenidos mediante identificación a partir de datos numéricos de entrada y salida, permite capturar la dinámica de la planta de forma muy fiel, incluso para sistemas complejos, muy cambiantes o desconocidos. Esta propiedad mejorará, lógicamente, la fidelidad de las predicciones y por tanto, en última instancia, mejorará el rendimiento del controlador predictivo.

Como conclusión final, resaltamos también el buen rendimiento de la estrategia mixta FMBPC/CLP, aplicada al control multivariable de procesos de fangos activados, un caso de estudio con un interés especial por su alta no linealidad y por la complejidad de la dinámica de la planta, debido a su carácter biológico.

En cuanto a posibles trabajos futuros, nos parece que sería interesante continuar y profundizar la investigación en los aspectos siguientes: a) influencia de los parámetros de cada una de las dos estrategias, FMBPC y CLP-MPC, en el comportamiento de las variables controladas, en el cumplimiento de las restricciones y en las características de la acción de control; b) aplicación de la estrategia propuesta a un caso de estudio con más restricciones, como restricciones en los incrementos de la acción de control (esfuerzos de control) u otros; c) utilización de modelos de predicciones alternativos en la estructura CLP-MPC, como modelos extendidos o aumentados, con los incrementos de la acción de control y/o con las perturbaciones; d) utilización de la estrategia propuesta en control MPC distribuido.

\section{Agradecimientos}

Este trabajo contó con el apoyo económico del Gobierno de España a través del proyecto MICINN PID2019-105434RB- 
C31 y de la Fundación Samuel Solórzano a través del proyecto FS / 20-2019.

\section{Referencias}

Adetola, V., \& Guay, M., 2010. Integration of real-time optimization and model predictive control. Journal of Process Control, 20(2), 125-133.

Al-Gherwi, W., Budman, H., Elkamel, A., 2013. A robust distributed model predictive control based on a dual-mode approach. Computers and Chemical Engineering, 50, 130-138.

Babuška, R., 1998a. Fuzzy Modeling for Control. Kluwer Academic Publishers, Boston, MA, USA.

Babuška, R., 1998b. Fuzzy Modeling and Identification Toolbox (FMID)User's Guide; Babuška, R., Delft, The Netherlands.

Blachini, F., 1999. Set invariance in control. Automatica, 35, 1747-1767.

Blažič, S., Škrjanc, I, 2007. Design and Stability Analysis of Fuzzy Modelbased Predictive Control-A Case Study. J. Intell. Robot. Syst., 49, 279 292. DOI: 10.1007/s10846-007-9147-8

Boulkaibet, I., Belarbi, K., Bououden, S., Marwala, T., Chadli, M., 2017. A new T-S fuzzy model predictive control for nonlinear processes. Expert Syst. Appl., 88, 132-151. DOI: 10.1016/j.eswa.2017.06.039

Bououden, S., Chadli, M., Karimi, H., 2015. An ant colony optimization-based fuzzy predictive control approach for nonlinear processes. Inf. Sci., 299, 143-158. DOI: 10.1016/j.ins.2014.11.050

Camacho, E. F., Bordons, C., 1998. Model Predictive Control. Springer, Great Britain.

El Bahja, H., 2017. Advanced control strategies based on invariance set theory and economic MPC: application to WWTP. Ph.D. Thesis, Universidad de Salamanca, Salamanca, Spain, 2017.

El Bahja, H., S.; Vega, P.; Revollar, S.; Francisco, M., 2018a. One Layer Nonlinear Economic Closed-Loop Generalized Predictive Control for a Wastewater Treatment Plant. Applied Sciences, 8(5), 657. DOI: 10.3390/app8050657

El Bahja, H., Vega, P., Tadeo, F., \& Francisco, M., 2018b. A constrained closed loop MPC based on positive invariance concept for a wastewater treatment plant. International Journal of Systems Science, 49(10), 2101 2115. DOI: $10.1080 / 00207721.2018 .1484195$

Francisco, M., Vega, P., 2006. Diseño Integrado de procesos de depuración de aguas utilizando control predictivo basado en modelos. RIAI-Revista Iberoamericana de Automática e Informática Industrial, 3(4), 88-98, ISSN 16977912

Gilbert, E.G., Tan, K. T., 1991. Linear systems with state and control constraints: the theory and application of maximal output admissible sets. IEEE Trans. AC, 36(9), 1008-1020.

Haber, R., Rossiter, J.A., and Zabet, K.R., 2016. An Alternative for PID control: Predictive Functional Control- A Tutorial. IEEE American Control Conference (ACC), 2016 (ACC2016). Boston, MA, USA, July 0608 .

Henze, M., Grady, C. P. L. Jr, Gujer, W., Marais, G. v. R., Matsuo, T., 1987. Activated Sludge Model No. 1. IAWPRC Scientific and Technical Reports No. 1. London, UK.

Limón, D., 2002. Control Predictivo de Sistemas no Lineales con Restricciones: Estabilidad y Robustez. Ph.D. Thesis, Universidad de Sevilla, Sevilla, Spain, 2002.

Lyapunov, A.M., 1892. The General Problem of the Stability of Motion (in Russian). Ph.D. Thesis, Kharkov Mathematical Society, Kharkov, Russia.
Lyapunov, A.M., 1992. The general problem of the stability of motion. Int. J. Control, 55, 531-534. DOI: 10.1080/00207179208934253

Maciejowski, J. M., 2002. Predictive Control with Constraints. Pearson Education Limited, Harlow, Essex, UK

Marchetti, A.G., Ferramosca, A. \& González, A.H., 2014. Steady-state target optimization designs for integrating real-time optimization and model predictive control. Journal of Process, 24 (1) 129-145.

Michalska, H., Mayne, D., 1993. Robust receding horizon control of constrained nonlinear systems. IEEE Transactions on Automatic Control, $38,1623-1633$

Mollov, S., Babuska, R., Abonyi, J., Verbruggen, H., 2004. Effective Optimization for Fuzzy Model Predictive Control. IEEE Trans. Fuzzy Syst., 12, 661-675. DOI: 10.1109/tfuzz.2004.834812

Moreno, R., 1994. Estimación de Estados y Control Predictivo de Proceso de Fangos Activados. Tesis Doctoral. Facultat de Ciències de la Universitat Autònoma de Barcelona (Spain).

Ramírez, K. J. , Gómez, L. M., Álvarez, H., 2014. Dual mode nonlinear model based predictive control with guaranteed stability. Ingeniería y Competitividad, 16(1), 23-34.

Richalet, J., 1993. Industrial application of model based predictive control. Automatica, 29 (5), 1251-1274.

Richalet, J., O’Donovan, D., 2009. Predictive Functional Control. Principles and Industrial Applications. Springer, London, UK.

Rossiter, J. A., 2003. Model-Based Predictive Control: A Practical Approach. CRC Press LLC, Boca Raton, Florida, EEUU.

Roubos, J., Mollov, S., Babuska, R., Verbruggen, H., 1999. Fuzzy modelbased predictive control using Takagi-Sugeno models. Int. J. Approx. Reason., 22, 3-30, doi:10.1016/s0888-613x(99)00020-1

Shariati, S., Noske, R., Brockhinke, A., Abel, D., 2015. Model predictive control of combustion instabilities using Closed-loop Paradigm with an incorporated Padé approximation of a phase shifter. 2015 European Control Conference (ECC). July 15-17. Linz, Austria.

Škrjanc, I., Matko, D., 2000. Predictive functional control based on fuzzy model for heat exchanger pilot plant. IEEE Transactions on Fuzzy Systems, 8 (6), 705-712.

Škrjanc, I., Blažič, S., 2016. Fuzzy Model-based Control - Predictive and Adaptive Approaches. In: Angelov, Plamen (Ed.), Handbook on Computational Intelligence. Vol. I. World Scientific, New Jersey, USA, Ch. 6, pp. 209-240. DOI: 10.1142/9789814675017_0006

Sorcia-Vázquez, F. D. J., Garcia-Beltran, C. D., Valencia-Palomo, G., Guerrero-Ramírez, G., Adam-Medina, M., Escobar-Jiménez, R., 2015. Control Predictivo Distribuido Óptimo Aplicado al Control de Nivel de un Proceso de Cuatro Tanques Acoplados. Revista Iberoamericana de Automática e Informática Industrial, 12, 365-375.

Takagi, T., Sugeno, M., 1985. Fuzzy Identification of Systems and its Application to Modeling and Control. IEEE Transactions on Systems, Man and Cybernetics, 15 (1), 116-132.

Vallejo, P. M., Vega, P., 2019. Analytical Fuzzy Predictive Control Applied to Wastewater Treatment Biological Processes. Complex., 2019, 5720185 doi:10.1155/2019/5720185

Vallejo, P. M., Vega, P., 2021. Practical Computational Approach for the Stability Analysis of Fuzzy Model-Based Predictive Control of Substrate and Biomass in Activated Sludge Processes. Processes, 9(3), 531. https://doi.org/10.3390/pr9030531

Zadeh, Lotfi A., 1990. Fuzzy Sets and Systems. International Journal of General Systems, 17 (2), 129-138. 